\title{
FAMILIES OF HOLOMORPHIC MAPS INTO RIEMANN SURFACES
}

BY

\author{
THEODORE J. BARTH(1)
}

\begin{abstract}
In analogy with the Hartogs theorem that separate analyticity of a function implies analyticity, it is shown that a separately normal family of holomorphic maps from a polydisk into a Riemann surface is a normal family. This contrasts with examples of discontinuous separately analytic maps from a bidisk into the Riemann sphere. The proof uses a theorem on pseudoconvexity of normality domains, which is proved via the following convergence criterion: a sequence $\left\{f_{j}\right\}$ of holomorphic maps from a complex manifold into a Riemann surface converges to a nonconstant holomorphic map if and only if the sequence $\left\{f_{j}^{-1}\right\}$ of set-valued maps, defined on the Riemann surface, converges to a suitable set-valued map. Extending Osgood's theorem, it is also shown that a separately analytic map (resp. a separately normal family of holomorphic maps) from a polydisk into a hyperbolic complex space is analytic (resp. normal).
\end{abstract}

1. A convergence criterion using divisors and level sets. By convention, all complex spaces (in particular complex manifolds) are connected and second countable; a Riemann surface is a 1-dimensional complex manifold.

We endow the family $A(M, N)$ of all holomorphic maps from the complex manifold $M$ into the complex space (usually a Riemann surface) $N$ with the compact-open topology [5, Definition 1.1, p. 257]. Convergence of sequences of holomorphic maps will always refer to this topology. Now $\mathrm{A}(M, N)$ may be regarded as a subspace of the space $C\left(M, N^{*}\right)$ of continuous maps from $M$ into the one-point compactification $N^{*}=N \cup\{\omega\}$ of $N$, again endowed with the compactopen topology. A sequence in $A(M, N)$ is said to diverge compactly if it converges to the map identically $\omega$ in $\mathrm{C}\left(M, N^{*}\right)[18, \mathrm{p} .197]$. The topological spaces $N$ and $N^{*}$ are metrizable. Equipping $N$ and $N^{*}$ with metrics, we see that convergence in $\mathrm{A}(M, N)$ and $\mathrm{C}\left(M, N^{*}\right)$ is equivalent to uniform convergence on com-

Received by the editors April 4, 1974.

AMS (MOS) subject classifications (1970). Primary 32A17, 32F15, 32H99; Secondary $32 \mathrm{H} 20$.

Key words and phrases. Nonnegative divisor, pseudoconvex domain, normal family of maps, normal family of divisors, normality domain, Hartogs theorem, Osgood theorem, hyperbolic complex space, Kobayashi distance.

(1) This work was done at Tulane University, while the author was on leave from the University of California, Riverside. 
pact sets [5, Theorem 7.2, p. 268] ; in turn this is equivalent to continuous convergence $[5,7.5$, pp. 268-269].

If $g$ is a holomorphic function defined on a neighborhood of a point $a$ in a complex manifold, we let $\nu(a, 0, g)$ denote the zero multiplicity (or total order) of $g$ at $a[15$, p. 156], [7, p. 8]. A function $\mu$, defined on a complex manifold $M$, is said to be a nonnegative divisor if each point $a$ in $M$ possesses an open connected neighborhood $U$ on which there is a holomorphic function $g$, not identically 0 , with $\nu(\cdot, 0, g)=\mu \mid U$. The support of the nonnegative divisor $\mu$ is supp $\mu=\{a \in M \mid \mu(a)>0\}$; it is either empty or an analytic set of pure codimension 1 in $M$. Stoll introduced the concept of convergence of a net of divisors $[15, p .176]$. In the special case which we use, his definition reduces to the following. A sequence $\left\{\mu_{j}\right\}$ of nonnegative divisors on the complex manifold $M$ is said to converge to the nonnegative divisor $\mu$ if for each point $a$ in $M$ there exist an open neighborhood $U$ of $a$, an integer $j_{0}$, and holomorphic functions $g_{j}\left(j \geqslant j_{0}\right)$ on $U$ satisfying

(a) $\nu\left(\cdot, 0, g_{j}\right)=\mu_{j} \mid U$ for $j \geqslant j_{0}$,

(b) $g_{j} \rightarrow g(j \rightarrow \infty)$

(c) $\nu(\cdot, 0, g)=\mu \mid U$;

in this case we write $\mu_{j} \rightarrow \mu(j \rightarrow \infty)$.

Hausdorff introduced a topology on the set of all closed subsets of a locally compact Hausdorff space $M[9, \S 28],[15$, pp. 196-199]. A point $a$ of $M$ is called a limit point of the sequence $\left\{F_{k}\right\}$ of closed subsets if there exist an integer $k_{0}$ and points $a_{k}$ in $F_{k}\left(k \geqslant k_{0}\right)$ such that $a=\lim a_{k}$; a point of $M$ is called a cluster point of $\left\{F_{k}\right\}$ if it is a limit point of some subsequence of $\left\{F_{k}\right\}$. If the set of limit points coincides with the set of cluster points, $\left\{F_{k}\right\}$ is said to converge to this set $F$, and we write $\lim F_{k}=F$ or $F_{k} \rightarrow F(k \rightarrow \infty)$.

Let $f$ be a nonconstant holomorphic map from the complex manifold $M$ into the Riemann surface $N$, and let $b$ be a point of $N$. Choose a map $\beta$ taking an open neighborhood $V$ of $b$ in $N$ biholomorphically onto an open subset of $\mathbf{C}$ and satisfying $\beta(b)=0$. The nonnegative integer

$$
\begin{aligned}
& \nu(a, b, f)=\nu(a, 0, \beta \circ f) \text { if } a \in f^{-1}(V), \\
& =0 \quad \text { if } a \in f^{-1}(N-V) \text {, }
\end{aligned}
$$

does not depend on the choice of $\beta$; it is called the b-multiplicity of $f$ at $a$. The function $v(\cdot, b, f)$ is a nonnegative divisor on $M$ with support $f^{-1}(b)$.

THEOREM 1. Let $\left\{f_{j}\right\}$ be a sequence of nonconstant holomorphic maps from the complex manifold $M$ into the Riemann surface $N$. Then the following are equivalent: 
(1) $\left\{f_{j}\right\}$ converges to a nonconstant map;

(2) there exist nonnegative divisors $\mu_{b}(b \in N)$ on $M$ such that \{supp $\mu_{b}$ \} is a partition of $M$ and $\nu\left(\cdot, b_{j}, f_{j}\right) \rightarrow \mu_{b}(j \rightarrow \infty)$ for every convergent sequence $b_{j} \rightarrow b(j \rightarrow \infty)$ in $N$;

(3) $M$ can be partitioned into closed proper subsets $M_{b}(b \in N)$ such that $f_{j}^{-1}\left(b_{j}\right) \rightarrow M_{b}(j \rightarrow \infty)$ for every convergent sequence $b_{j} \rightarrow b(j \rightarrow \infty)$ in $N$.

Proof. $(1) \Rightarrow(2)$. Assume that $\left\{f_{j}\right\}$ converges to a nonconstant map $f$. Define $\mu_{b}=\nu(\cdot, b, f)$ for $b \in N$. Clearly $\left\{\operatorname{supp} \mu_{b}\right\}=\left\{f^{-1}(b)\right\}$ is a partition of $M$. Let $b_{j} \rightarrow b(j \rightarrow \infty)$ be a convergent sequence in $N$. Fix a point $a$ of $M$. In case $f(a) \neq b$, there is an open neighborhood $U$ of $a$ such that $b \notin f(U)$. Shrinking $U$, we can find an integer $j_{0}$ such that $b_{j} \notin f_{j}(U)$ for $j \geqslant j_{0}$. Let $g$, $g_{j}\left(j \geqslant j_{0}\right)$ be the constant function identically 1 on $U$; then all divisors involved are identically 0 . In case $f(a)=b$, choose a map $\beta$ taking an open neighborhood $V$ of $a$ in $N$ biholomorphically onto an open subset of $\mathbf{C}$ and satisfying $\beta(b)=0$. There exist an index $j_{0}$ such that $b_{j} \in V$ for $j \geqslant j_{0}$ and a neighborhood $U$ of $a$ such that $f(U) \subset V$. Shrinking $U$ and increasing $j_{0}$ if necessary, we may assume that $f_{j}(U) \subset V$ for $j \geqslant j_{0}$. Set $g=\beta \circ f \mid U$, and $g_{j}=\beta \circ\left(f_{j} \mid U\right)-\beta\left(b_{j}\right)$ for $j \geqslant j_{0}$. In either case $(f(a) \neq b$ or $f(a)=b)$, we have

$$
\begin{gathered}
\nu\left(\cdot, 0, g_{j}\right)=\nu\left(\cdot, b_{j}, f_{j}\right) \mid U \text { for } j \geqslant j_{0}, \\
g_{j} \rightarrow g(j \rightarrow \infty) \text { and } v(\cdot, 0, g)=\nu(\cdot, b, f)\left|U=\mu_{b}\right| U .
\end{gathered}
$$

Thus $\nu\left(\cdot, b_{j}, f_{j}\right) \rightarrow \mu_{b}(j \rightarrow \infty)$.

(2) $\Rightarrow$ (3). Taking $M_{b}=\operatorname{supp} \mu_{b}$, this follows from [15, Theorem 4.10, pp. 199-200].

(3) $\Rightarrow(1)$. Assume that (3) holds. If $\left\{f_{j}\right\}$ converges, the limit map must be nonconstant, for otherwise (3) would imply that $M_{b}=\varnothing$ for all $b$ in $N$ except the value of the limit map. Equip $N$ with a complete metric inducing its topology [5, Corollary 2.4, pp. 294-295]. Suppose $\left\{f_{j}\right\}$ does not converge. Then there is a compact subset $K$ of $M$ such that $\left\{f_{j}\right\}$ does not converge uniformly on $K$. By the Cauchy criterion, there exist $\epsilon>0$, subsequences $\left\{g_{k}{ }^{\prime}\right\}$ and $\left\{h_{k}\right\}$ of $\left\{f_{j}\right\}$, and points $a_{k}$ in $K$ such that $d\left(g_{k}\left(a_{k}\right), h_{k}\left(a_{k}\right)\right) \geqslant \epsilon$ for all $k$. By taking a subsequence, we may assume that $a_{k} \rightarrow a(k \rightarrow \infty)$. By (3), $a \in M_{b}$ for some (uniquely determined) $b$ in $N$. Let $V$ be a compact neighborhood of $b$ in $N$ having diameter less than $\epsilon$. By taking a subsequence and exchanging the roles of $\left\{g_{k}\right\}$ and $\left\{h_{k}\right\}$ if necessary, we may assume that $h_{k}\left(a_{k}\right) \notin V$ for all $k$. By (3), $f_{j}^{-1}(b) \rightarrow M_{b}(j \rightarrow \infty) ;$ hence $h_{k}^{-1}(b) \rightarrow M_{b}(j \rightarrow \infty)$. Since $a \in M_{b}=$ 
$\lim h_{k}^{-1}(b)$, there exist an integer $k_{0}$ and points $c_{k}$ in $h_{k}^{-1}(b)\left(k \geqslant k_{0}\right)$ such that $a=\lim c_{k}$. Choose open connected subsets $U_{k}\left(k \geqslant k_{0}\right)$ of $M$ such that $a_{k}, c_{k}$ $\in U_{k}$ and $\left\{U_{k}\right\}$ is a base for the neighborhood system of $a$. Now $h_{k}\left(c_{k}\right)=b \in V$ and $h_{k}\left(a_{k}\right) \notin V$; since $h_{k}$ is continuous and $U_{k}$ is connected, there exists a point $d_{k}$ in $U_{k}$ such that $h_{k}\left(d_{k}\right) \in \partial V$. Of course $d_{k} \rightarrow a(k \rightarrow \infty)$; taking a subsequence, we may assume that $e_{k}=h_{k}\left(d_{k}\right) \rightarrow e \in \partial V(k \rightarrow \infty)$. By (3) again, $h_{k}^{-1}\left(e_{k}\right) \rightarrow M_{e}(k \rightarrow \infty)$. Thus $a=\lim d_{k} \in \lim h_{k}^{-1}\left(e_{k}\right)=M_{e}$. Since $b \neq e$, we have $M_{b} \cap M_{e}=\varnothing$, a contradiction.

This proof shows how the limit map $f$ determines the nonnegative divisors $\mu_{b}=\nu(\cdot, b, f)$ and the closed sets $M_{b}=f^{-1}(b)$. We could have simplified the statement of Theorem 1 by introducing a holomorphic map $f$ and using the divisors and closed sets determined by it. The present statement is easier to use (for example in Theorem 2), since one need not know a priori that the candidate for the limit map is holomorphic. We note that the implication (3) $\Rightarrow(1)$ works for continuous maps from a locally connected locally compact Hausdorff space into a locally compact metric space. Using similar techniques, we can characterize compact divergence.

THEOREM 1'. Let $\left\{f_{j}\right\}$ be a sequence of nonconstant holomorphic maps from the complex manifold $M$ into the Riemann surface $N$. Then the following are equivalent:

(1) $\left\{f_{j}\right\}$ diverges compactly;

(2) $\nu\left(\cdot, b_{j}, f_{j}\right) \rightarrow 0(j \rightarrow \infty)$ for every convergent sequence $b_{j} \rightarrow b$ $(j \rightarrow \infty)$ in $N$;

(3) $f_{j}^{-1}\left(b_{j}\right) \rightarrow \varnothing(j \rightarrow \infty)$ for every convergent sequence $b_{j} \rightarrow b$ $(j \rightarrow \infty)$ in $N$.

2. Pseudoconvexity of normality domains. A Hartogs figure in $\mathbf{C}^{m}$ is a pair $(H, P)$ with

$$
\begin{aligned}
H= & \left\{w \in \mathbf{C}^{m}|| w_{j}\left|<r, 1 \leqslant j \leqslant m-1 ; s^{\prime}<\right| w_{m} \mid<s\right\} \\
& \cup\left\{w \in \mathbf{C}^{m}|| w_{j}\left|<r^{\prime}, 1 \leqslant j \leqslant m-1 ;\right| w_{m} \mid<s\right\}, \\
A= & \left\{w \in \mathbf{C}^{m}|| w_{j}|<r, 1 \leqslant j \leqslant m-1 ;| w_{m} \mid<s\right\},
\end{aligned}
$$

where $0<r^{\prime}<r, 0<s^{\prime}<s$. An open subset $M_{0}$ of the $m$-dimensional complex manifold $M$ is said to be pseudoconvex in $M$ if $\tau(A) \subset M_{0}$ whenever $(H, A)$ is a Hartogs figure and $\tau: A \rightarrow \tau(A) \subset M$ is a biholomorphic map satisfying $\tau(H) \subset$ $M_{0}$. We follow the standard practice of ignoring the biholomorphic map $\tau$ and acting as if $H$ and $A$ are subsets of $M$.

We now recall a few important properties of a Hartogs figure $(H, A)$. By 
the Cauchy integral formula, every holomorphic function on $H$ extends (uniquely) to a holomorphic function on $A$. By extending its reciprocal, we see that the extension of a nowhere zero holomorphic function on $H$ has no zeros in $A$. It follows that a holomorphic function on $H$ and its extension to $A$ have exactly the same image set. Since a nonempty analytic set of pure codimension 1 in $A$ is the zero set of a holomorphic function on $A$ [7, Lemma 12, p. 251], such a set must intersect $H$. Finally, if $\lambda$ and $\mu$ are nonnegative divisors on $A$ that agree on $H$, we can invoke Cousin II [7, Theorem 6, p. 249] to obtain holomorphic functions $g$ and $h$ on $A$ such that $\nu(\cdot, 0, g)=\lambda$ and $\nu(\cdot, 0, h)=\mu$; the meromorphic function $g / h$ is holomorphic and without zeros on $H$, hence on $A$, and it follows that $\lambda=\mu$.

A family $F$ of holomorphic maps from the complex manifold $M$ into the complex space $N$ is called normal if every sequence in $F$ has a subsequence that either converges or diverges compactly [6, Definition 3, p. 114], [18, Definition 1.1, p. 197]. A family $N$ of nonnegative divisors on the complex manifold $M$ is called normal if every net in $N$ has a convergent subnet [15, p. 154]; in fact $N$ is normal if and only if every sequence in $N$ has a convergent subsequence [15, Theorem 2.24, p. 188]. The normality domain of the family $F$ (resp. $N$ is the union of the open connected subsets $U$ of $M$ such that the restriction $F \mid U$ (resp. $N \mid U)$ is normal. Since normality is a local property, the restriction of a family to any open connected subset of its normality domain is a normal family. Julia [11] proved that the normality domain of a family of holomorphic functions is pseudoconvex, i.e., he proved that the normality domain of $F$ is pseudoconvex in $M$ in case $N=$ C. In this section we show this holds for any Riemann surface $N$. The uniformization theorem and [3, Theorem 2, p. 296] give this for any Riemann surface $N$ not biholomorphic to the Riemann sphere, but we shall not need this result. We shall use the fact, proved in [2], that the normality domain of $N$ is pseudoconvex in $M$; of course we can interpret this pseudoconvexity result as an extension theorem.

Lemma 1. Let $(H, A)$ be a Hartogs figure, and let $\left\{\mu_{j}\right\}$ be a sequence of nonnegative divisors on $A$. If $\left\{\mu_{j} \mid H\right\}$ converges (as a sequence of nonnegative divisors on $H)$, then $\left\{\mu_{j}\right\}$ converges.

Proof. Let $N=\left\{\mu_{j} \mid j=1,2,3, \ldots\right\}$. By hypothesis, $H$ is contained in the normality domain of $N$. The pseudoconvexity of this normality domain [2, Theorem 12.4, p. 245] shows that $N$ is a normal family. Thus $\left\{\mu_{j}\right\}$ has a subsequence converging to a nonnegative divisor $\mu$ on $A$. If $\left\{\mu_{j}\right\}$ does not converge to $\mu$, there is a subnet $\left\{\lambda_{t}\right\}$ of $\left\{\mu_{j}\right\}$ such that no subnet of $\left\{\lambda_{t}\right\}$ converges to $\mu[15$, Lemma 2.27, p. 191]. Since $N$ is a normal family, we may 
assume that $\left\{\lambda_{t}\right\}$ converges to a nonnegative divisor $\lambda$ on $A$. The divisors $\lambda$ and $\mu$ agree on $H$, hence $\lambda=\mu$, a contradiction.

Lemma 2. Let $\lambda_{j} \rightarrow \lambda(j \rightarrow \infty)$ and $\mu_{j} \rightarrow \mu(j \rightarrow \infty)$ be convergent sequences of nonnegative divisors on the complex manifold $M$. If (supp $\left.\lambda_{j}\right) \cap$ $\left(\right.$ supp $\left.\mu_{j}\right)=\varnothing$ for all $j$, then $(\operatorname{supp} \lambda) \cap($ supp $\mu)$ is either empty or an analytic set of pure codimension 1 in $M$.

Proof. If the analytic set $X=(\operatorname{supp} \lambda) \cap(\operatorname{supp} \mu)$ is nonempty, take a point $a$ in $X$. By the definition of convergence in the space of divisors, there exist an open connected neighborhood $U$ of $a$, an integer $j_{0}$, and holomorphic functions $f_{j}, g_{j}\left(j \geqslant j_{0}\right)$ on $U$ such that

$$
\begin{gathered}
\nu\left(\cdot, 0, f_{j}\right)=\lambda_{j}\left|U, \quad \nu\left(\cdot, 0, g_{j}\right)=\mu_{j}\right| U \text { for } j \geqslant j_{0}, \\
f_{j} \rightarrow f, \quad g_{j} \rightarrow g(j \rightarrow \infty), \\
\nu(\cdot, 0, f)=\lambda|U, \quad \nu(\cdot, 0, g)=\mu| U .
\end{gathered}
$$

Now $f(a)=g(a)=0, f \not 0 \not \equiv g$; thus the product $f g$ has total order $k$ at $a$, where $1<k<\infty$. By [7, Lemma 2, p. 13] or [17, Lemma $4 \mathrm{H}$, p. 13], there is a coordinate system $\left(w_{1}, \cdots, w_{m-1}, z\right)$ about $a=0$ such that $f g$ is regular of order $k$ in $z$. Shrinking $U$, we may assume that $f(0, z) g(0, z) \neq 0$ if $z \neq 0$ and $(0, z)$ $\in U$.

There exist an open connected neighborhood $W$ of 0 in $\mathbf{C}^{m-1}$ and an open disk $\Delta$ around 0 in $\mathrm{C}$ such that $W \times \Delta$ is a relatively compact subset of $U$ and $f$ and $g$ are good for $W \times \Delta[17$, Definition 5B, pp. 14-15]. Shrinking $W$ and increasing $j_{0}$, we may assume that $f_{j}$ and $g_{j}$ are good for $W \times \Delta$ if $j \geqslant j_{0}$. For $w$ in $W$, let $p$ (resp. $q, p_{j}, q_{j}$ ) denote the number of zeros of $f(w, \cdot)$ (resp. $\left.g(w, \cdot), f_{j}(w, \cdot), g_{j}(w, \cdot)\right)$ in $\Delta$ (counting multiplicities); by [17, Lemma $5 \mathrm{E}$, p. 15], these integers do not depend on the choice of $w$. In view of their integral representation $[17,(5.2), \mathrm{p} .15], p_{j} \rightarrow p, q_{j} \rightarrow q(j \rightarrow \infty)$. Thus we may assume that $p_{j}=p, q_{j}=q$ if $j \geqslant j_{0}$. Using the Weierstrass preparation theorem, we uniquely factor

$$
\begin{array}{ll}
f \mid W \times \bar{\Delta}=F P, & f_{j} \mid W \times \bar{\Delta}=F_{j} P_{j}, \\
g \mid W \times \bar{\Delta}=G Q, & g_{j} \mid W \times \bar{\Delta}=G_{j} Q_{j},
\end{array}
$$

so that $P, P_{j}$ (resp. $Q, Q_{j}$ ) are Weierstrass polynomials of degree $p$ (resp. $q$ ) in $z$ and $F, F_{j}, G, G_{j}$ are holomorphic functions without zeros on $W \times \bar{\Delta}\left(j \geqslant j_{0}\right)$. By the Newton identities $[16$, p. 81$]$, the coefficients in each Weierstrass poly- 
nomial are polynomials in the power sums of the roots; in turn, as in [17, pp. 16-17], these power sums can be expressed as integrals involving the factored function. It follows that $P_{j} \rightarrow P, Q_{j} \rightarrow Q(j \rightarrow \infty)$ in $A(W \times \mathrm{C}, \mathrm{C})$. Let $R$ denote the resultant operator on polynomials of formal degrees $p$ and $q$ [16, pp. 83-84], [17, p. 342]. Define

$$
\begin{aligned}
& h(w)=R(P(w, \cdot), Q(\dot{w}, \cdot)), \\
& h_{j}(w)=R\left(P_{j}(w, \cdot), Q_{j}(w, \cdot)\right) .
\end{aligned}
$$

Then $h, h_{j} \in A(W, C)$, and $h_{j} \rightarrow h(j \rightarrow \infty)$. Since $\left(\operatorname{supp} \lambda_{j}\right) \cap\left(\operatorname{supp} \mu_{j}\right)=\varnothing$, $P_{j}$ and $Q_{j}$ have no common zeros in $W \times \Delta$, hence in $W \times \mathbf{C}$. By the basic property of resultants, the functions $h_{j}$ have no zeros. On the other hand, $P(0)=Q(0)=0$, so $h(0)=0$. By a classical theorem of Hurwitz, $h \equiv 0$. Thus, for every point $w$ in $W$, $P(w, \cdot)$ and $Q(w, \cdot)$ have a common zero in $\Delta$. Let $\pi: \mathbf{C}^{m-1} \times \mathbf{C} \rightarrow \mathbf{C}^{m-1}$ denote the projection. We have just proved that the light holomorphic map $\pi \mid X \cap(W \times \Delta): X \cap(W \times \Delta) \rightarrow W$ is onto; it follows that this map takes each neighborhood of $a=0$ in $X$ onto a neighborhood of 0 in $W$. By [17, Theorem 6B(b), p. 122], $\operatorname{dim}_{a} X=\operatorname{dim}_{0} W=m-1$.

THEOREM 2. Let $F$ be a family of holomorphic maps from the complex manifold $M$ into the Riemann surface $N$. Then the normality domain of $F$ is pseudoconvex in $M$.

Proof. Let $M_{0}$ denote the normality domain of $F$. Ignoring the implicit biholomorphic map, we take a Hartogs figure $(H, A)$ satisfying $A \subset M$ and $H \subset$ $M_{0}$; we must prove that $A \subset M_{0}$. Let $\left\{h_{k}\right\}$ be a sequence in F. Since $H \subset M_{0}$, there is a subsequence $\left\{f_{j}\right\}$ of $\left\{h_{k}\right\}$ that either diverges compactly or converges on $H$.

If $\left\{f_{j}\right\}$ diverges compactly on $H$, implication (1) $\Rightarrow(2)$ of Theorem $1^{\prime}$ shows that $\nu\left(\cdot, b_{j}, f_{j}\right) \mid H \rightarrow 0(j \rightarrow \infty)$ for every convergent sequence $b_{j} \rightarrow b$ $(j \rightarrow \infty)$ in $N$. By Lemma 1 , we may replace $H$ with $A$ in the last clause. Implication $(2) \Rightarrow(1)$ of Theorem $1^{\prime}$ completes the proof that $\left\{f_{j}\right\}$ diverges compactly on $A$.

If $\left\{f_{j} \mid H\right\}$ converges to a constant map with value $b$, we will prove that $\left\{f_{j} \mid A\right\}$ also does. By slimming the Hartogs figure, we may assume that $\left\{f_{j}\right\}$ converges on an open set containing the closure of $H$. Let $V$ be an open neighborhood of $b$ that is biholomorphically equivalent to an open set in $\mathbf{C}$. Then $V \supset$ $f_{j}(H)=f_{j}(A)$ for $j$ sufficiently large. Hence $\left\{f_{j} \mid A\right\}$ converges to a constant map with value $b$.

If $\left\{f_{j} \mid H\right\}$ converges to a nonconstant map $g$, we may assume that $f_{j}$ is nonconstant for all $j$. Let $b_{j} \rightarrow b(j \rightarrow \infty)$ be a convergent sequence in $N$. By 
implication $(1) \Rightarrow(2)$ of Theorem $1, \nu\left(; b_{j}, f_{j}\right) \mid H \rightarrow \nu(;, b, g)(j \rightarrow \infty)$; by Lemma 1 , there is a nonnegative divisor $\mu_{b}$ on $A$ such that $\nu\left(; b_{j}, f_{j}\right) \mid A \rightarrow \mu_{b}$ $(j \rightarrow \infty)$. Since $\mu_{b} \mid H=\nu(\cdot, b, g)$, the divisor $\mu_{b}$ depends only on the point $b$ and not on the particular sequence $b_{j} \rightarrow b(j \rightarrow \infty)$ used in its definition. We now show that the family supp $\mu_{b}$ \} is a partition of $A$. If $b$ and $c$ are distinct points of $N$,

$$
\begin{aligned}
& \left(\operatorname{supp} v\left(\cdot, b, f_{j}\right) \mid A\right) \cap\left(\operatorname{supp} v\left(\cdot, c, f_{j}\right) \mid A\right) \\
& \quad=A \cap f_{j}^{-1}(b) \cap f_{j}^{-1}(c)=\varnothing \quad \text { for all } j .
\end{aligned}
$$

By Lemma 2, the analytic set $X=\left(\operatorname{supp} \mu_{b}\right) \cap\left(\operatorname{supp} \mu_{c}\right)$ is either empty or has pure codimension 1 in $M$. But $X \cap H=g^{-1}(b) \cap g^{-1}(c)=\varnothing$, hence $X=\varnothing$, proving that $\left\{\right.$ supp $\mu_{b}$ \} is disjoint. To see that this family covers $A$, we fix a point $a$ in $A$ and define $d_{j}=f_{j}(a)$. Then $a \in f_{j}^{-1}\left(d_{j}\right)=\operatorname{supp} \nu\left(\cdot, d_{j}, f_{j}\right)$. Since the pure 1-codimensional analytic set $f_{j}^{-1}\left(d_{j}\right)$ contains the point $a$ of $A$, it intersects $H$; the convergence of $\left\{f_{j}\right\}$ on $H$ then implies the convergence of a subsequence of $\left\{d_{j}\right\}$ to a point $d$ in $N$. Using Lemma 1 and implication (1) $\Rightarrow$ (2) of Theorem 1 , we see that the corresponding subsequence of $\left\{\nu\left(\cdot, d_{j}, f_{j}\right) \mid A\right\}$ converges to a nonnegative divisor $\mu$ on $A$. The divisors $\mu$ and $\mu_{d}$ agree on $H$, hence on $A$. Thus $a \in \operatorname{supp} \mu=\operatorname{supp} \mu_{d}$. By implication (2) $\Rightarrow(1)$ of Theorem $1,\left\{f_{j} \mid A\right\}$ converges.

3. A Hartogs theorem for normal families of maps. A classical theorem of Hartogs asserts that a separately holomorphic function is holomorphic [8], [10, Theorem 2.2.8, pp. 28-29]. Let $D$ denote the open unit disk $\{z \in \mathbf{C l}$ $|z|<1\}$. A coordinate disk in the polydisk $D^{m}$ is a submanifold of the form $\left\{\left(d_{1}, \cdots, d_{k-1}\right)\right\} \times D \times\left\{\left(d_{k+1}, \cdots, d_{m}\right)\right\}$ where $d_{j} \in D$. A map from a polydisk $D^{m}$ into a complex space is said to be separately holomorphic if its restriction to each coordinate disk is holomorphic. In precise terms, the Hartogs theorem says that if $N=\mathrm{C}$, every separately holomorphic map $f: D^{m} \rightarrow N$ is holomorphic. In view of the loose statement that "separate analyticity implies analyticity," it is natural to expect that the conclusion of the Hartogs theorem holds for an arbitrary complex space (or at least complex manifold) $N$. However, such a theorem fails if $N=\mathrm{C} \cup\{\infty\}$ is the Riemann sphere: the map $f: D^{2} \rightarrow$ $\mathrm{C} \cup\{\infty\}$ defined by

$$
\begin{aligned}
f(w, z) & =(w+z)^{2} /(w-z) \text { if } w \neq z, \\
& =\infty \quad \text { if } \quad w=z \neq 0, \\
& =0 \quad \text { if } \quad w=z=0,
\end{aligned}
$$


is separately holomorphic but is not continuous at $(0,0)$. Of course the problem lies in continuity. If $f$ is known to be continuous, $N$ may be replaced by a domain in $\mathbf{C}^{n}$, and separate analyticity implies analyticity by Osgood's lemma [7, Theorem 2, pp. 2-3]. We shall use the following generalized version of the Hartogs theorem.

LEMMA 3. Let $N$ be a complex manifold whose universal covering manifold is Stein. Then every separately holomorphic map $f: D^{m} \rightarrow N$ is holomorphic.

Proof. Proceeding by induction on $m$, we may assume that every separately holomorphic map from $D^{m-1}$ into $N$ is holomorphic. Since the conclusion is local, it is enough to prove that $f$ is holomorphic in a neighborhood of 0 . Let $U$ be a neighborhood of $f(0)$ in $N$ that is biholomorphically equivalent to an open set in $\mathbf{C}^{n}$, and let $V$ be an open neighborhood of $f(0)$ whose closure is contained in $U$. Regard $D^{m}$ as $D^{m-1} \times D$. By hypothesis, $f(0, \cdot): D \rightarrow N$ is continuous; hence there is an open disk $\Delta$ centered at 0 such that $f(\{0\} \times \Delta) \subset V$. Take $z$ in $\Delta$; by the induction assumption, $f(\cdot z): D^{m-1} \rightarrow N$ is continuous; thus there exists $r(z)>0$ such that $f(w, z) \in V$ if $\left|w_{j}\right|<r(z)(j=1, \cdots, m-1)$. This means that the union of the sets $B(k)=\left\{z \in \Delta \mid f(w, z) \in V\right.$ if $\left|w_{j}\right|<1 / k(j=1$, $\cdots, m-1)\}(k=1,2, \cdots)$ is $\Delta$. By the Baire category theorem, there is an integer $k_{0}$ such that the closure of $B\left(k_{0}\right)$ contains a nonempty, open, connected, simply connected set $Z$. Let $W=\left\{w \in \mathbf{C}^{m-1}|| w_{j} \mid<1 / k_{0}\right\}$. If $(w, z) \in W \times Z$, $z$ is a limit of points $z^{\prime}$ in $B\left(k_{0}\right)$ satisfying $f\left(w, z^{\prime}\right) \in V$; the continuity of $f(w, \cdot)$ shows that $f(w, z) \in \bar{V} \subset U$. By Osgood's theorem [10, Lemma 2.2.9, p. 28], [14], $f \mid W \times Z: W \times Z \rightarrow U \subset N$ is holomorphic. Let $p: L \rightarrow N$ be the universal covering manifold of $N$. Since $W \times Z$ is simply connected, there exists a holomorphic map $g: W \times Z \rightarrow L$ such that $p \circ g=f \mid W \times Z$. Choose a point $z_{0}$ in $Z$, and fix it for the rest of this proof. For each $w$ in $W$, the holomorphic map $f(w, \cdot): D \rightarrow N$ can be lifted to a holomorphic map $h(w, \cdot): D \rightarrow L$ with $p \circ h(w, \cdot)=f(w, \cdot)$ and $h\left(w, z_{0}\right)=g\left(w, z_{0}\right)$; by uniqueness of lifting, $h(w, z)=$ $g(w, z)$ if $z \in Z$. Using the imbedding theorem for Stein manifolds [7, Theorem 13, p. 226], we may regard $L$ as a complex submanifold of a euclidean space $\mathbf{C}^{q}$. Consider the map

$$
h: W \times D \rightarrow L \subset C^{q} ;
$$

$h|W \times Z=g| W \times Z$ is holomorphic and $h(w, \cdot): D \rightarrow L$ is holomorphic for each $w$ in $W$. Applying the Hartogs lemma [10, Lemma 2.2.11, pp. 28-29] to its coordinates, we see that $h$ is holomorphic. Thus $p \circ h=f \mid W \times D$ is holomorphic.

A family of holomorphic maps from a polydisk into a complex space is 
said to be separately normal if its, restriction to each coordinate disk is normal. Nishino has proved a normal families version of the Hartogs theorem: a separately normal family of holomorphic functions is normal [13, Theorème II, pp. 263-264]. In view of the failure of the Hartogs theorem for a single map into the Riemann sphere, it is somewhat surprising that Nishino's theorem extends to Riemann surfaces.

THEOREM 3. If $F$ is a separately normal family of holomorphic maps from a polydisk $D^{m}$ into a Riemann surface $N$, then $F$ is a normal family.

PROOF. By the uniformization theorem, the universal covering surface of $N$ is the Riemann sphere, $\mathrm{C}$, or $D$.

Assume that the universal covering surface of $N$ is the Riemann sphere. Then up to biholomorphic equivalence, $N=\mathrm{C} \cup\{\infty\}$. Let $\left\{f_{j}\right\}$ be a sequence in $F$. If $f_{j} \equiv \infty$ for infinitely many $j$, we can extract a subsequence converging to the constant map identically $\infty$. Otherwise, there is a subsequence $\left\{g_{k}\right\}$ of $\left\{f_{j}\right\}$ such that $g_{k} \not \equiv$ for all $k$. Now $G=\left\{g_{k} \mid k=1,2,3 \cdots\right\}$ may be regarded as a separately normal family of meromorphic functions on $D^{m}$; by [13, Theorème IV, pp. 279-282], this family is normal as a family of meromorphic functions. Extracting a subsequence, we may assume that $\left\{g_{k}\right\}$ converges as a sequence of meromorphic functions on $D^{m}$, i.e., $[13, \mathrm{p} .273]$ there is an analytic set $S$ of codimension at least 2 in $D^{m}$ such that $\left\{g_{k} \mid D^{m}-S\right\}$ converges in $\mathrm{A}\left(D^{m}-S, N\right)$. Thus $D^{m}-S$ is contained in the normality domain of $G$; by Theorem 2 , this normality domain must equal $D^{m}$. It follows that a subsequence of $\left\{f_{j}\right\}$ converges.

Assume that the universal covering surface of $N$ is $\mathbf{C}$ or $D$. Let $\left\{f_{j}\right\}$ be a sequence in $F$. There exists a subsequence $\left\{g_{k}\right\}$ of $\left\{f_{j}\right\}$ such that $\left\{g_{k}(0)\right\}$ converges to a point of $N$ (or to $\omega$ in the one-point compactification of $N$ ). If infinitely many of the maps $g_{k}$ are constant, it is easy to extract a convergent (resp. compactly divergent) subsequence; thus we may assume that $g_{k}$ is nonconstant for all $k$. Write $D^{m}=D^{m-1} \times D$. Extracting a subsequence, we may assume that $\left\{g_{k}(0, \cdot)\right\}$ converges (resp. diverges compactly) in $A(D, N)$. Let $E$ be a countable dense subset of $D$. Proceeding by induction on $m$, we may assume that the restriction of $F$ to each polydisk $D^{m-1} \times\{z\}(z \in D)$ is a normal family. Extracting successive subsequences and taking a diagonal, we may assume that $\left\{g_{k}(; z)\right\}$ converges (resp. diverges compactly) in $A\left(D^{m-1}, N\right)$ for every $z$ in $E$. Take $w$ in $D^{m-1}$; since $\left\{g_{k}(w, \cdot)\right\}$ consists of maps in a normal family and converges pointwise in $N$ (resp. to $\omega$ ) on the dense subset $E$ of $D$, this sequence converges (resp. diverges compactly) in $A(D, N)$. Similarly, $\left\{g_{k}(\cdot, z)\right\}$ converges (resp. diverges compactly) in $A\left(D^{m-1}, N\right)$ for each $z$ in $D$. Define 
$g: D^{m} \rightarrow N \cup\{\omega\}$ by $g(w, z)=\lim g_{k}(w, z)$. Either $g\left(D^{m}\right) \subset N$ or $g \equiv \omega$; in the first case we conclude from Lemma 3 that $g$ is holomorphic. Suppose no subsequence of $\left\{g_{k}\right\}$ converges to $g$ in $\mathrm{C}\left(D^{m}, N^{*}\right)$. Then there exists a convergent sequence $a_{k} \rightarrow a(k \rightarrow \infty)$ in $D^{m}$ such that $g_{k}\left(a_{k}\right) \nrightarrow g(a)(k \rightarrow \infty)$. Extracting a subsequence we may assume that $b_{k}=g_{k}\left(a_{k}\right) \rightarrow b(k \rightarrow \infty)$, where $g(a) \neq b \in N^{*}$. There is a convex open neighborhood $G$ of $a$ in $D^{m}$ such that $b \notin g(G)$. (If $g \equiv \omega$ or any other constant, we may even take $G=D^{m}$.) Let $V_{k}=g_{k}^{-1}\left(b_{k}\right) \cap G$. The sequence $\left\{V_{k}\right\}$ consists of pure $(m-1)$-dimensional analytic sets in $G$. If $H$ is a coordinate disk in $D^{m}, g_{k}|H \cap G \rightarrow g| H \cap G$ $(k \rightarrow \infty)$ in $C\left(H \cap G, N^{*}\right)$; hence $\left\{V_{k} \cap H\right\}$ is a locally finite sequence. By [1, Theorem 4, pp. 339-340], $\left\{V_{k}\right\}$ is locally finite. But for $k$ sufficiently large, $a_{k} \in V_{k}$ and $a_{k} \rightarrow a(k \rightarrow \infty)$, a contradiction.

4. Maps into higher dimensional complex manifolds. In [3, Theorem 2, p. 296] it was shown that the normality domain of a family of holomorphic maps from an $m$-dimensional complex manifold $M$ into an $n$-dimensional complex manifold $N$ is $(m-n)$-pseudoconvex in $M$, provided the universal covering manifold of $N$ is Stein. In view of Theorem 2, it seems that the condition on the universal covering manifold may be superfluous. Unfortunately, Theorem 1 does not have an obvious generalization to maps into higher dimensional complex manifolds, so an attempt at a proof along the same lines seems futile.

The statement of Theorem 3 becomes false if the words "Riemann surface" are replaced by "complex manifold." Take $F=\left\{f_{j} \mid j=1,2, \cdots\right\}$ where $f_{j}: D^{2}$ $\rightarrow \mathrm{C}^{2}$ is defined by $f_{j}(w, z)=j^{2}\left(w-j^{-1}, z-j^{-1}\right)$. The restriction of $\left\{f_{j}\right\}$ to each coordinate disk diverges compactly, hence $F$ is separately normal. But $f_{j}\left(j^{-1}, j^{-1}\right)=(0,0)$, so $F$ cannot be a normal family. The difficulty here is caused by the relative dimensions. To handle this, we define an $n$-dimensional coordinate polydisk in $D^{m}(1 \leqslant n \leqslant m)$ to be the cartesian product of $n$ copies of $D$ and $m-n$ singleton subsets of $D$. The following theorem can be proved using the technique in the last paragraph of the proof of Theorem 3. Again, it seems that the assumption about the universal covering manifold may be unnecessary.

THEOREM 4. Let $F$ be a family of holomorphic maps from a polydisk $D^{m}$ into a complex manifold of dimension $n \leqslant m$ whose universal covering manifold is Stein. If the restriction of $F$ to each n-dimensional coordinate polydisk in $D^{m}$ is a normal family, then $F$ is a normal family.

Finally, we note that strong Hartogs-type theorems hold for maps into (Kobayashi) hyperbolic complex spaces [12, pp. 97-98]. Since these spaces may be regarded as generalizations of the unit disk, the following might more 
properly be called an Osgood-type theorem [14].

Theorem 5. Let $N$ be a hyperbolic complex space. Then

(1) every separately holomorphic map $f: D^{m} \rightarrow N$ is holomorphic;

(2) every separately normal family $F$ of holomorphic maps from $D^{m}$ into $N$ is normal.

Proof. (1) By Osgood's lemma [7, Theorem 2, pp. 2-3], it will suffice to prove that $f$ is continuous. Using the triangle inequality and the fact that holomorphic maps from $D$ into $N$ are distance decreasing with respect to the Poincare distance $\rho$ on $D$ and the Kobayashi distance $d_{N}$ on $N$, we obtain the inequality $d_{N}(f(z), f(a)) \leqslant \Sigma \rho\left(z_{j}, a_{j}\right)$ for all $z$ and $a$ in $D^{m}$. Thus $f$ is continuous as a map of $D^{m}$ into the metric space $\left(N, d_{N}\right)$. Since $d_{N}$ induces the standard topology on $N$ [4], $f$ is continuous.

(2) Let $\left\{f_{j}\right.$ \} be a sequence in $F$. As in the beginning of the last paragraph of the proof of Theorem 3, we can find a subsequence $\left\{g_{k}\right\}$ of $\left\{f_{j}\right\}$ such that $g(z)=\lim g_{k}(z) \in N^{*}$ exists for all $z$ in $D^{m}$. Either $g\left(D^{m}\right) \subset N$ or $g \equiv \omega$; in the first case we use (1) to conclude that $g$ is holomorphic. The inequality $d_{N}\left(g_{k}(z), g_{k}(a)\right) \leqslant \Sigma \rho\left(z_{j}, a_{j}\right)$ and pointwise convergence of $\left\{g_{k}(a)\right\}$ to $g(a)$ now imply the continuous convergence of $\left\{g_{k}\right\}$ to $g$.

\section{BIBLIOGRAPHY}

1. H. Alexander, B. A. Taylor and J. L. Ullman, Areas of projections of analytic sets, Invent. Math. 16 (1972), 335-341. MR 46 \#2078.

2. T. J. Barth, Families of nonnegative divisors, Trans. Amer. Math. Soc. 131 (1968), 223-245. MR 36 \#2830.

3. - Normality domains for families of holomorphic maps, Math. Ann. 190 (1971), 293-297. MR 43 \#3486.

4. - The Kobayashi distance induces the standard topology, Proc. Amer. Math. Soc. 35 (1972), 439-441. MR 46 \#5668.

5. J. Dugundji, Topology, Allyn and Bacon, Boston, Mass., 1966. MR 33 \#1824.

6. H. Grauert and H. Reckziegel, Hermitesche Metriken and normale Familien holomorpher Abbildungen, Math. Z. 89 (1965), 108-125. MR 33 \#2827.

7. R. C. Gunning and H. Rossi, Analytic functions of several complex variables, Prentice-Hall, Englewood Cliffs, N. J., 1965. MR 31 \#4927.

8. F. Hartogs, Zur Theorie der analytischen Funktionen mehrerer unabhängiger Veränderlichen, insbesondere über die Darstellung derselben durch Reihen, welche nach Potenzen einer Veränderlichen fortschreiten, Math. Ann. 62 (1906), 1-88.

9. F. Hausdorff, Mengenlehre, 3rd ed., de Gruyter, Berlin, 1935; photographic reproduction, Dover, New York, 1944. MR 7, 419.

10. L. Hörmander, An introduction to complex analysis in several variables, Van Nostrand, Princeton, N. J., 1966. MR 34 \#2933.

11. G. Julia, Sur les familles de fonctions analytiques de plusieurs variables, Acta Math. 47 (1926), 53-115.

12. S. Kobayashi, Hyperbolic manifolds and holomorphic mappings, Pure and Appl. Math. 2, Marcel Dekker, New York, 1970. MR 43 \#3503.

13. T. Nishino, Sur une propriété des familles de fonctions analytiques de deux variables complexes, J. Math. Kyoto Univ. 4 (1965), 255-282. MR 31 \#3632. 
14. W. F. Osgood, Note über analytische Funktionen meherer Veränderlichen, Math. Ann. 52 (1899), 462-464.

15. W. Stoll, Normal families of non-negative divisors, Math. Z. 84 (1964), 154-218. MR 29 \#2431.

16. B. L. van der Waerden, Modern Algebra. Vol. 1, Springer, Berlin, 1937; English transl., Ungar, New York, 1949. MR 10, 587.

17. H. Whitney, Complex analytic varieties, Addison-Wesley, Reading, Mass., 1972.

18. H. Wu, Normal families of holomorphic mappings, Acta Math. 119 (1967), 193-233. MR 37 \#468.

DEPARTMENT OF MATHEMATICS, UNIVERSITY OF́ CALIFORNIA, RIVERSIDE, CALIFORNIA 92502

DEPARTMENT OF MATHEMATICS, TULANE UNIVERSITY, NEW ORLEANS, LOUISIANA 70118 